\title{
Producción de materiales en lámina delgada mediante técnicas láser
}

\author{
C.N. Afonso ${ }^{(*)}$
}

\begin{abstract}
Resumen Mediante la moderna técnica de depósito por láser pulsado (PLD) se pueden obtener materiales de alto interés tecnológico, como son óxidos complejos o cerámicas duras que no son fáciles de obtener por otras técnicas más convencionales. En el presente trabajo, se revisan, en primer lugar, las características especiales de esta técnica. A continuación, se presentan los resultados obtenidos en las investigaciones actualmente en marcha en nuestro laboratorio sobre dopado óptico de materiales mediante especies activas, como los iones de tierras raras o partículas nanocristalinas en matrices aislantes.
\end{abstract}

Palabras clave: Depósito por láser pulsado. Ablación por láser.

\section{Production of thin films by means of pulsed laser deposition}

\begin{abstract}
Pulsed laser deposition is a recently developed technique with a large potential in producing materials of technological interest that are not easily produced by more conventional techniques, such as complex oxides or hard ceramics. We will first review the special features of this technique to then show current results of our own research in producing optically doped waveguides either with rare-earth ions or metal nanoparticles.
\end{abstract}

Keywords: PLD. Laser ablation.

\section{RESUMEN AMPLIADO}

Cuando un haz láser suficientemente intenso se focaliza sobre la superficie de un blanco se producen diversos fenómenos, tanto de naturaleza puramente térmica como electrónica, que dan lugar a la eyección de material del blanco en forma de electrones, especies atómicas, especies diatómicas y/o agregados. La densidad y grado de excitación de estas especies en la superficie del blanco es muy elevada, lo que se manifiesta en la aparición de una "pluma" visible que se expande en la dirección perpendicular al blanco. Si se coloca una superficie (substrato) en la trayectoria de expansión de la misma, una fracción de las especies se adhiere a ella dando lugar al crecimiento de una lámina delgada. Aun cuando esta idea es conocida prácticamente desde la invención del láser de rubí en los años sesenta, no se ha utilizado de forma extensiva para la producción de materiales en lámina delgada hasta finales de los años ochenta, cuando se demostró que las mejores láminas superconductoras de

(*) Instituto de Óptica (CSIC). Serrano 121. 28006-Madrid (España). alta temperatura crítica (HTCS) conocidas hasta la fecha podían producirse por medio de esta técnica, haciendo uso de una atmósfera de oxígeno durante el crecimiento. En ese momento se inicia una actividad científica importante encaminada no sólo a entender los mecanismos físicos que posibilitan la calidad de sus resultados, sino también a explorar su aplicación a otros materiales, especialmente aquellos en los que técnicas mas convencionales no producen resultados satisfactorios, como son los óxidos complejos. Si bien la técnica fue inicialmente conocida como ablación láser, el nombre más comúnmente utilizado en la actualidad es el de depósito por láser pulsado (PLD). Se ha intentado sintetizar de forma gráfica su evolución y relevancia en la figura 1 donde se representa, separadamente, el número de trabajos publicados en HTCS y en otros materiales, ampliando la gráfica publicada anteriormente (1). Puede verse claramente cómo ambas curvas siguen inicialmente una tendencia similar. No obstante, a partir de 1992 se produce un incremento considerable de la actividad en materiales diferentes de los HTCS, mientras que la actividad, en estos últimos, decae a un valor que se mantiene casi constante. La actividad en otros materiales estuvo, en un principio, 


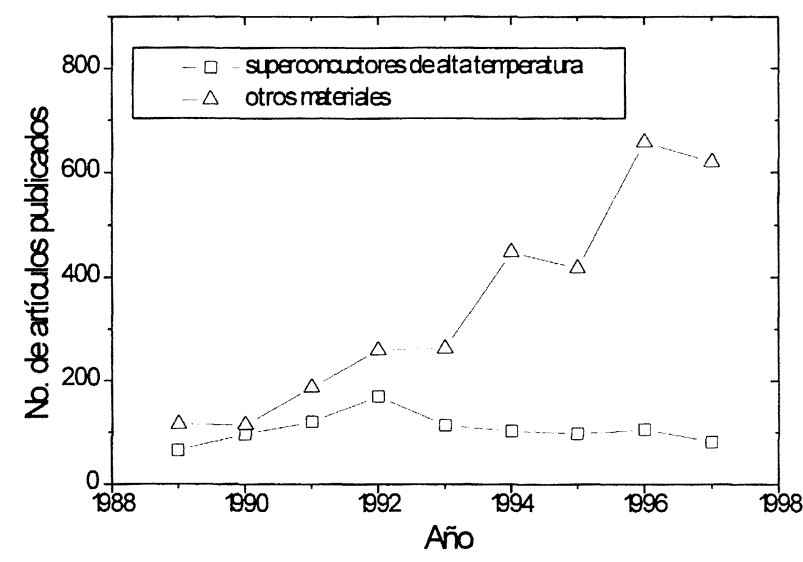

FIG. 1.- Número de trabajos publicados por año en ablación láser y PLD desde 1989.

\section{FIG. 1.- Number of papers published per year on PLD and laser ablation since 1989.}

concentrada fundamentalmente en la producción de óxidos cerámicos, aunque, en seguida, se exploraron otros materiales de interés tecnológico, tales como materiales ultraduros, biocompatibles, metales, semiconductores y, más recientemente, materiales nanoestructurados. Muchos de los aspectos que hacen que la técnica de PLD sea tan atractiva, así como una revisión bibliográfica sobre los materiales producidos por ella, se encuentran reunidos en un libro publicado recientemente sobre PLD (2).

El objetivo de esta conferencia es, por un lado, dar una visión general de algunas de las propiedades que hacen de la PLD una técnica única a la hora de producir materiales en los que otras técnicas fracasan y, por otro lado, presentar la tendencia actual de nuestra actividad en este campo que está centrada en la producción de guías de onda dopadas ópticamente. Dentro del primer aspecto, discutiremos el carácter cuasi-estequiométrico del proceso de ablación, la existencia de especies energéticas tanto neutras como ionizadas $(10-100 \mathrm{eV})$ y las elevadas velocidades de depósito accesibles $\left(10^{4} \mathrm{~nm} / \mathrm{s}\right)$. En particular, la influencia de estas especies energéticas en las propiedades de las láminas es especialmente crítica y es posible modificarla mediante la utilización de un gas que altere la cinética de expansión de las especies o cambios en la configuración blanco-substrato. Como ejemplo del efecto que la presencia de estas especies energéticas tiene en las propiedades de láminas y, en particular, en la producción de recubrimientos ópticos de gran adherencia y alta densidad, se muestra en la figura 2 la densidad óptica de láminas de germanio amorfo de diferentes espesores obtenidas por tres técnicas diferentes (depósito por haz de electrones, pulverización catódica y PLD) y tomando como referencia una lámina gruesa obtenida por pulverización catódica. Como puede verse, las láminas obtenidas por

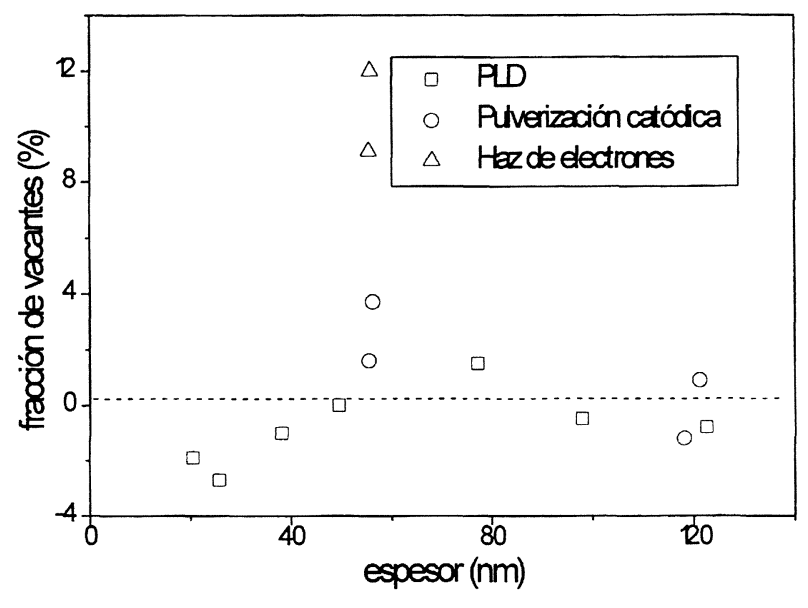

FIG. 2.- Fracción de vacantes en láminas de germanio amorfo crecidas por tres técnicas diferentes y para diferentes espesores de las láminas. Como referencia, se ha tomado una lámina gruesa obtenida por pulverización catódica.

FIG. 2.- Void fraction of amorphous Ge films grown by three different techniques, namely, PLD, sputtering and electron beam deposition. The origin of the vertical axis corresponds to a thick film grown by sputtering.

PLD, aunque próximas a las obtenidas por pulverización catódica, son las más densas, llegando incluso a ser más densas que la de referencia (3).

Actualmente nuestra actividad en PLD se centra en el "dopado óptico de materiales", término que utilizamos para referirnos al proceso de incorporación de especies activas en matrices, de forma que el conjunto presente una actividad óptica especial. En particular, estamos interesados en la incorporación de iones de tierra-rara o partículas nanocristalinas en matrices aislantes. En el primer caso, los iones de tierra-rara conservan su estructura electrónica y, por tanto, sus bandas de emisión, independientemente de la matriz en la que se sumergen. El interés de dichas bandas de emisión es bien conocido desde hace bastantes años, en particular por su aplicación para el desarrollo de láseres y amplificadores. Los ejemplos comerciales más conocidos son el láser de Nd-YAG y el amplificador de erbio (erbio en vidrio). Este último ha revolucionado el campo de las comunicaciones ópticas al permitir amplificar señales ópticas de forma directa, evitando la conversión de señal óptica-eléctrica-óptica en el proceso de amplificación (4). Las partículas nanocristalinas metálicas embebidas en matrices aislantes, dan lugar a bandas de absorción características (5). Este hecho se conoce desde hace siglos y se ha utilizado ampliamente para embellecer las catedrales con hermosas vidrieras. Ya en este siglo, estas bandas de absorción han posibilitado el desarrollo de filtros coloreados, gafas de sol o 
ventanas selectivas. Más recientemente se ha sabido que estos materiales nanoestructurados presentan, además, excelentes propiedades ópticas no lineales con un tiempo de respuesta en la escala temporal de los picosegundos y estando, por tanto, entre los candidatos más prometedores para el desarrollo de conmutadores ópticos ultrarrápidos. Uno de los retos actuales más importantes en el campo del dopado óptico es el de encontrar una tecnología adecuada para desarrollar estos materiales en lámina delgada/guía de onda. En este contexto, la técnica de PLD parece la más adecuada, ya que las matrices más idóneas son óxidos complejos. Los resultados obtenidos por nosotros en guías de onda de $\mathrm{Er}_{2} \mathrm{Al}_{2} \mathrm{O}_{3}$ (6) y $\mathrm{Cu}: \mathrm{Al}_{2} \mathrm{O}_{3}$ (7) obtenidas por PLD son excelentes y, como ejemplo de ello, se muestra en la figura 3 una

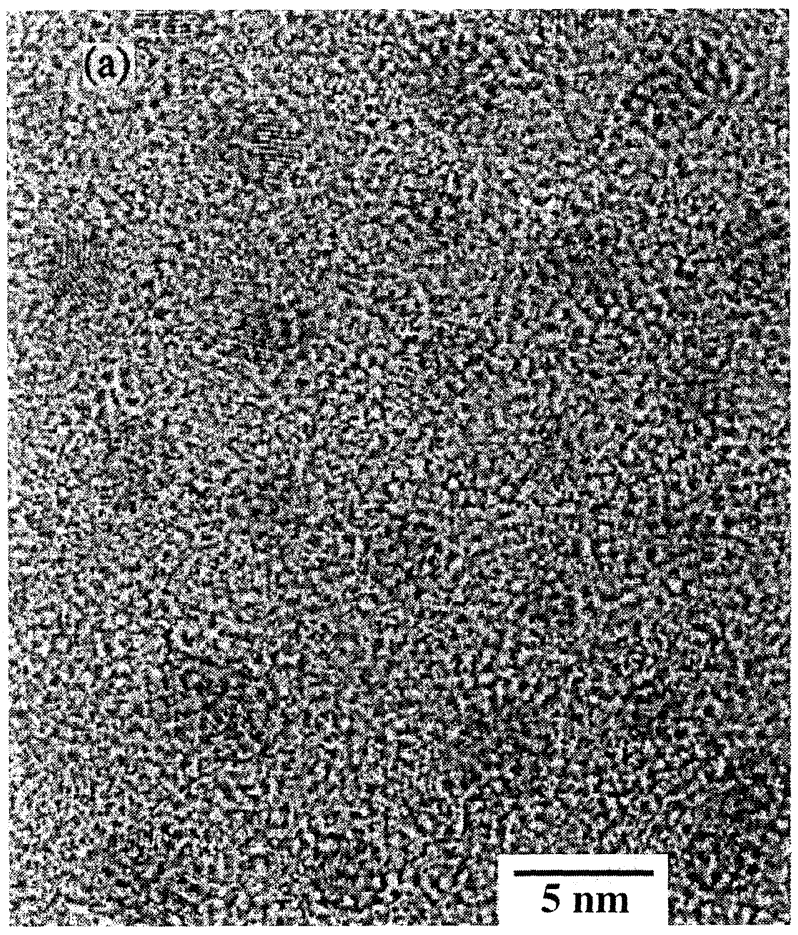

imagen obtenida por microscopía electrónica de alta resolución de una lámina de $\mathrm{Cu}: \mathrm{Al}_{2} \mathrm{O}_{3}$ (8). En la misma se aprecia un conjunto de zonas oscuras de unos pocos nanometros con líneas interferenciales de los planos reticulares del cobre y que corresponden a los nanocristales. A su lado se muestra un histograma con el diámetro de estos nanocristales en el que puede observarse que la distribución es muy estrecha. Si se compara este tipo de distribución con la que se obtiene con uno de los métodos más utilizados en la actualidad para producir el dopado óptico por nanopartículas, la implantación iónica (9), se observa cómo la técnica de PLD ofrece una distribución mucho más homogénea. Las propiedades ópticas no lineales de estas láminas están entre las mejores publicadas hasta la fecha (8).

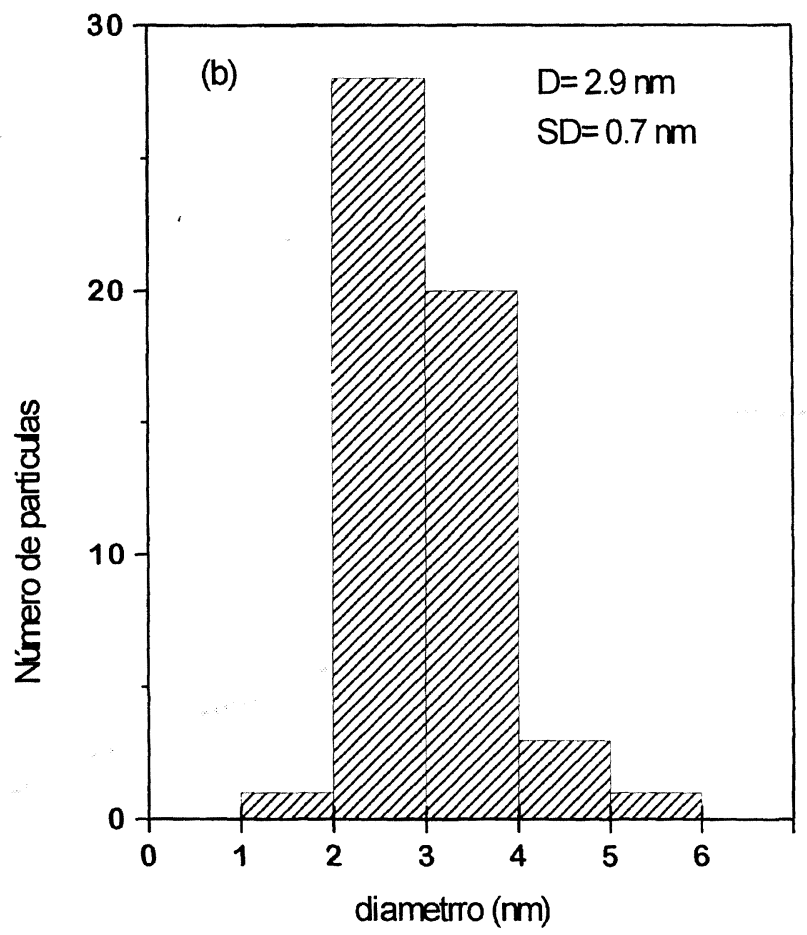

FIG. 3.- - a) Imagen obtenida por microscopía electrónica de transmisión de alta resolución en una muestra de $\mathrm{Cu}: \mathrm{Al}_{2} \mathrm{O}_{3}$ preparada por PLD en la que las zonas oscuras corresponden a los nanocristales de cobre. $b$ ) Histograma del diámetro de los nanocristales de cobre obtenidos de la imagen en $a$ ).

FIG. 3.- a) High resolution transmission electron microscopy image of a $\mathrm{Cu}: \mathrm{Al}_{2} \mathrm{O}_{3}$ film prepared by $\mathrm{PLD}$, the dark contrast areas being the copper nanocrystals. b) Histogram of the copper nanocrystals diameter obtained from an extended area of the image in a).

\section{REFERENCIAS}

(1) Afonso, C.N. "Pulsed laser deposition of films for optical applications" in Insulating materials for optoelectronics: new developments, editado por F. Agulló-López, World Scientific Publishing Co. Inc, Cap. 1 (1995)

(2) Pulsed laser deposition of thin films, de CHRISEY, D.B. and Hubler, G.K. John Wiley \& Son, Inc, NY. (1994)

(3) De Sande, J.C.G., Afonso, C.N., Escudero, J.L., Serna, R., Catalina, F., Bernabeu, E. Applied Optics 31, 6133 (1992)

(4) Desurvire, E. Er-doped fiber amplifiers: principles and applications, John Wiley \& Sons, Inc., New York (1994)
(5) Optical properties of metal clusters, KREIBIG, U. VOLLMER, M. Springer-Verlag (1995)

(6) Serna, R., Afonso, C.N. Appl. Phys. Lett. 69, 1541 (1996)

(7) Ballesteros, J.M., Serna, R., Solis, J., Afonso, C.N., Petford-Long, A.K., Osborne, D.H., Haglund, R.F. Jr., Appl. Phys. Lett. 71, 2445 (1997)

(8) Afonso, C.N., Serna, R., Ballesteros, J.M., Solis, J SPIE Proceedings, 6th International Conference on advanced laser technologies, Limoges (1997)

(9) Mazzoldi, P., Arnold, G.W., Battaglin, G., Bertoncello, R., Gonella, F. Nucl. Instr. and Meth. B91, 478 (1994) 